\title{
Electrokinetics of Temperature for Development and Treatment of Effusions
}

\author{
Oliver Szasz ${ }^{1}$, Gyula Peter Szigeti², Attila Marcell Szasz ${ }^{3}$ \\ ${ }^{1}$ Department of Biotechnics, Istvan University, Budaors, Hungary \\ ${ }^{2}$ Institute of Human Physiology and Clinical Experimental Research, Semmelweis University, Budapest, Hungary \\ ${ }^{3}$ Cancer Center, Semmelweis University, Budapest, Hungary \\ Email: biotech@gek.szie.hu
}

How to cite this paper: Szasz, O., Szigeti, G.P. and Szasz, A.M. (2017) Electrokinetics of Temperature for Development and Treatment of Effusions. Advances in Bioscience and Biotechnology, 8, 434-449.

https://doi.org/10.4236/abb.2017.811032

Received: October 7, 2017

Accepted: November 26, 2017

Published: November 29, 2017

Copyright $\odot 2017$ by authors and Scientific Research Publishing Inc. This work is licensed under the Creative Commons Attribution International License (CC BY 4.0).

http://creativecommons.org/licenses/by/4.0/

\begin{abstract}
Introduction: Hyperthermia is a complementary therapy in oncology having various pros and contras for its application. Ascites, pleural effusion, edema and other electrolyte accumulations are frequently excluded from the treatability of the patients with heating locally or systemically. The special gathering of electrolytes is sometimes contraindicated, at times not mentioned in the clinical protocols. However, it is certainly challenging in the oncology where micro and macro edemas, as well as larger electrolyte accumulations (e.g. ascites, pleural effusion), are very frequent. Methods: Excluding patients with accumulation of free electrolytes limits the applications of hyperthermia. To find a solution we are studying the microvasculature and fluid dynamism together with the electric field effects, including the injury currents. The hyperthermia method which we investigate is the modulated electro-hyperthermia $(\mathrm{mEHT})$. We use the Starling's equation and the injury current in the frame of non-equilibrium thermodynamics and in connection with the biologically closed electric circuits. Results: It is shown that mEHT, unlike the conventional hyperthermia, is applicable for patients who have edema and other free-electrolytes in the volume which is targeted. The heterogeneous heating (unlike the homogeneous, isothermal conventional hyperthermia) promotes the development of tumor-specific immune actions, and so has less adverse-effects, and longer survival time for patients in advanced, metastatic cancers too. Conclusion: $\mathrm{mEHT}$ is well applicable in cases of ascites, pleural effusion, edema and other electrolyte accumulations when a patient is treated in complex (complementary) oncological therapy.
\end{abstract}

\section{Keywords}

Effusion, Ascites, Edema, Modulated Electro-Hyperthermia, mEHT 


\section{Introduction}

One of the repeated questions connected to oncological treatments is the challenge of free electrolytes (effusions like cerebral-edema, ascites, pleuritis, pericarditis). It is serious: "Malignant pleural effusion complicates the care of approximately 150,000 people in the United States each year" [1]. Hyperthermia is a complementary therapy in oncology, and it frequently has doubts having many pros and contras for its application. Free electrolytes are sometimes contraindicated, sometimes not mentioned in the clinical protocols. However, it is certainly challenging in oncology where micro and macro effusions, as well as larger electrolyte accumulations (e.g. ascites, pleural effusion, cerebral edema), occur frequently. Excluding patients with free electrolytes (like marked ascites [2]) limits the application of hyperthermia also in the cases where it would be clinically indicated.

Effusions are a swelling in micro or macro sizes. In the event of injury or inflammation, the regular body response is frequently effusion. Effusion is a fluid from the blood vessels that allows more fighting to enter the affected area and supplies the healing processes with extra energy, too. Many special conditions could cause an effusion. Homeostatic mechanisms regulate the fluid levels in the body. The capillary vessel is a semipermeable filter. The system has hydrostatic pressures of the capillary vessels and the interstitial fluid around it, as well as the osmotic pressures on both sides of the separating cell-wall. The processes have to be taken into account in forming the effusion:

1) The filtration, which is responsible for fluid efflux from the vessel and in reverse;

2) The reabsorption;

3) The influx to the vessel;

4) The diffusion which is simply guided by the concentration gradients;

5) The pinocytosis (fluid endocytosis), which makes membrane "bubbles" and could form isolated vesicles encompassing fluid inside.

Multiple reasons form free electrolytes:

1) Hydrostatic pressure increases,

2) Lymphatic drainage is obstructed,

3) Oncotic pressure decreases in the vessel,

4) Oncotic pressure increases in interstitial fluid,

5) Permeability of the vessel-wall increases,

6) Retention of water is in the tissues (e.g. dysfunction of kidney).

Hyperthermia is a local or systemic heating of the body. We know that systemic hyperthermia could cause swelling in the brain, and the brain edema is life-threatening [3]. Hyperthermia could be applied for various physiotherapies, but it is the most complex application for the treatment of cancer. Numerous kinds of heating methods exist for oncologic hyperthermia from very local ablation techniques through the locally focused electromagnetic waves to the whole-body heating [4] [5]. Some local and whole-body oncologic hyperthermia treatments cause 
brain edema [6] [7], starting at $42^{\circ} \mathrm{C}$ [8].

There are special hyperthermia combinations exist for peritoneal effusions [9]. These are surgery in combination with hyperthermic intraperitoneal chemotherapy (HIPEC) which could be done by laparoscopy or open surgery, as well [10]. In this article we do not study the surgery-combined methods, only the non-invasive technics are considered. One special technique of oncologic hyperthermia is the modulated electro-hyperthermia (mEHT; trade name oncothermia) [11]. Its effects on effusions like malignant ascites are not cleared yet. Our present objective is to clarify the effect of mEHT on a broad range of free electrolytes, (including such sensitivities than brain edema), and a lot of body fluids (like ascites, pleural effusion, etc., but of course excluding the normal physiologically free electrolytes, e.g. urine).

\section{Materials and Methods}

The method of mEHT has definite inhomogeneous heating using the membrane specialties of the malignant cells [12]. It affects microvasculature nearby by elevated temperature, but only in the mild (fever) range [13]. The method has a strong synergy of the electric field and temperature [14], and shows clinical advantages [15]. These results are based on some theoretical considerations, which are presented in this article together with other supportive clinical trials with mEHT.

The production of interstitial fluid can be well approached by forces in Starling's equation. The Starling's equation describes the fluid balance across the wall of a capillary-vessel, considering the hydrostatic pressure gradient and the oncotic (osmotic) pressure gradient across the capillary. There are four forces counted, two-two for the capillary and its interstitial fluid:

1) Hydrostatic pressure in the capillary $\left(p_{c}\right)$;

2) Hydrostatic pressure in the interstitial fluid $\left(p_{i}\right)$;

3) The oncotic pressure in the capillary $\left(\pi_{c}\right)$;

4) The oncotic pressure in the interstitial fluid $\left(\pi_{i}\right)$.

The Starling's equation using these forces is [16]

$$
J_{v}=C_{f}\left[\left(p_{c}-p_{i}\right)-\sigma\left(\pi_{c}-\pi_{i}\right)\right]
$$

where $C_{f}$ is the filtration coefficient, and $\sigma$ is the reflection coefficient. $C_{f}$ indicates the capillary permeability depending on the capillary surface and its hydraulic conductance. The $\sigma$ reflection coefficient is a correction factor, which is necessary for the usually very selective permeability of the vessel-wall. It is used to correct the value of the gradient by large molecules. It is near to 1 when no protein could cross the wall, and near to zero when everything goes through, there is no real filtration of the wall. Conventionally, the sign of the forces chosen positive when it directs outward, and negative for the inward force. When (1) is positive the electrolyte flows out from the capillary, while when it is negative, the fluid enters absorbed by the capillary. In the normal homeostasis, the filtration directs out from the capillary in all along the vessel, not changes at the 
arteriole-venule transition zone [17]. However, the whole phenomena with the lymph interaction are more complex [18], definite, unchanged direction in every situation could not be given, the system is homeostatic feedback regulated [19]. Furthermore, the Starling's equation is not accurate enough for complete physiological processes at the micro-fluid exchange in capillary level. Probably glycocalyx has an additional effect which modifies the Starling's forces [20] [21].

In the case of effusion connected to injury or cancer, an additional transport forms. Due to the local polarization of epithelial cells, in the event of any disorder, it induces charge transfer named injury-current (IC) [22]; which has a major role in the healing of the tissue [23].

This charge-transfer is an electric current responsible for numerous physiological changes in the microenvironment of the injury [24]. Typical values of IC are around $100 \mu \mathrm{A} / \mathrm{cm}^{2}$ having approx. $100 \mathrm{mV} / \mathrm{cm}$ electric field (EF) and extended to the mm-range distance from the wound [25]. The induced EF is pointing to the wound area, and IC has a closed loop through the wound and the surface of the epithelium. It is measured with high-tech methods at the wound healing process [26] [27]. EF controls the healing process and persists till the wound exists. It controls the cell-division [28], directs the cell migration [29], with exponentially decreasing the EF to zero within $3 \mathrm{~mm}$ [30] to heal the wound. The wound healing by external EF stimulation is also known [31].

The charge transports in aqueous electrolytes create a mass-transport of water molecules. The electric current induces electrolyte flow and vice versa. The phenomenology of this process can be described by the theory of Onsager's non-equilibrium thermodynamics [32]. Two forces have a role in this process, the change of electric potential $\left(\Delta_{\varphi}\right)$ and the hydrodynamical pressure $\left(\Delta_{p}\right)$. These induce initiate electric $\left(J_{e}\right)$ and mass $\left(J_{m}\right)$ currents, which are interacting. $J_{e}$ is the IC due to the polarization effects [23], and $J_{m}$ could be the flow of electrolyte due to the various pressure differences [33]. The theory assumes linear interaction between these quantities, including the cross effect which is also linear. The following set of equations is:

$$
\begin{aligned}
& J_{v}=L_{11} \Delta p+L_{12} \Delta \varphi, \\
& J_{e}=L_{21} \Delta p+L_{22} \Delta \varphi
\end{aligned}
$$

The $L_{i k}$ Onsager's phenomenological constants are symmetric, and due to the entropy-law, the constants of the true effects are positive. The mixed indexed coefficients could be negative also, but according to the experimental data, they are always considerably lower than the main effects, so negligible in most of the cases. Therefore:

$$
L_{12}=L_{21}, L_{11}>0, L_{22}>0, L_{11} L_{22} \geq L_{12}^{2}
$$

It is realistic to assume that the extracellular space has constant pressure, every change is quickly equalized, so according to (2):

$$
J_{v}=\frac{L_{12}}{L_{22}} J
$$


Hence, the $J_{m}$ and $J_{e}$ could not necessarily flow in the same direction. Realistic quantitative assumptions are $J_{e}=10 \mathrm{nA}$ and $L_{12} / L_{22}=10^{-4}$. In this case:

$$
J_{v}=\frac{L_{12}}{L_{22}} J=10^{-11} \mathrm{~m}^{3} / \mathrm{s}=36 \mathrm{~mm}^{3} / \mathrm{h}
$$

This is considerable value to deliver water during the IC flow making an influence on cells and transports.

When we apply heating and increase the temperature in the targeted tissue, the heat-flow and the temperature-gradient should be considered. Then instead of (2) we have additional terms and equation to the Onsager's constitutive equations, a new current (heat-current $J_{q}$ ) must be added and driven by the $\Delta T$ temperature gradient and $T$ changes in the hyperthermia-range $\left(37^{\circ} \mathrm{C}-45^{\circ} \mathrm{C}\right)$ only:

$$
\begin{aligned}
& J_{v}=L_{11} \Delta p+L_{12} \Delta \varphi+L_{13} \Delta T, \\
& J=L_{21} \Delta p+L_{22} \Delta \varphi+L_{23} \Delta T, \\
& J_{q}=L_{31} \Delta p+L_{32} \Delta \varphi+L_{33} \Delta T
\end{aligned}
$$

The $L_{i k}$ phenomenological coefficients are constant, and the symmetry, as well as the positivity (entropy) conditions, are:

$$
\begin{aligned}
& L_{12}=L_{21}, L_{13}=L_{31}, L_{23}=L_{32}, \\
& L_{11}>0, L_{22}>0, L_{33}>0, \\
& L_{11} L_{22} \geq L_{12}^{2},\left|\begin{array}{lll}
L_{11} & L_{12} & L_{13} \\
L_{21} & L_{22} & L_{23} \\
L_{31} & L_{32} & L_{33}
\end{array}\right|>0
\end{aligned}
$$

The third factor of the micro-environment of the wound is the concept of "biologically closed electric circuits" (BCEC) [34] [35], which states interacting current loops in the tissues, which could act both micro- and macro-scales [36]. The argument of BCEC is centered on the electrolyte transports, which is charge-transport at the same time. However, in the media, where multiple semipermeable barriers (membranes) and the bidirectional flows of the electrophoretic-dielectrophoretic system of electrolytes modify the simple mechanical electrolyte circulation and net electric-current can be measured. The malignant tissue has a certain potential gradient to its healthy neighborhood [34] [37], which acts to promote and direct the cancer-cell migration [38], very similar to the IC concept in wound cases. A cancer treatment was developed [39] [40] using BCEC principles. These types of methods apply outside the electric field to generate currents and have been found effective against cancer [41] [42] [43].

The Vascular-Interstitial Closed Circuit (VICC) is one kind of BCEC process [44]. The circulations of various electrolytes like lymph, blood, and extracellular-matrix induce electric currents form various closed circuits, electric current-loops. VICC is activated and permanently powered by metabolic energy and the IC. The chronic VICC may induce pathological processes, and vice versa, the neoplastic formation can be healed by artificially constrained electrophoretic forces. The BCEC idea was so popular that even a society was formed to study it [45]. The complex biologic electric microcircuits promoted by BCEC probably could 
be extended and completed with hydrodynamic microcirculation for special transport of drugs.

It is a long-time hypothesis that cancer is a wound which does not heal, [46]. After a long "dormant" status of this hypothesis, nowadays it is revised [47], showing evidence about the similarities of the wound and cancer [48] [49] [50]; and a deeper research was started regarding stem cells [51] and with chronic fibrosis added to the cancer-wound parallelism [52] [53]. It is shown that wound promotes the epidermal tumorigenesis [54], which shows the possible connection between the wound and cancer again. Theoretically based [55], and measured [56] the link of the injury currents with neoplastic processes; and recent research shows "how tumors hijack body's wound healing process" [57].

\section{Results}

One of the therapies with curative intent of cancer is oncologic hyperthermia. A tumor (like the wound) could have naturally higher temperature than its environment; the higher metabolic rate could increase the local temperature [58]. IC does not make temperature increase in the wound because its low power (approx. $0.01 \mathrm{~mW} / \mathrm{g}$ ) does not increase the local heat-production [59].

When therapeutic hyperthermia is applied, energy is absorbed by the target from the outside sources. Both the electric field and the temperature affect the transport of the water and influence the development of effusion. When the temperature of the target increases, the resting potential of the cells will be more negative with the temperature coefficient $-(0.2 \div 0.3) \mathrm{mV} / \mathrm{K}$. In the case of the $44^{\circ} \mathrm{C}$ target-temperature, $1.5-2 \mathrm{mV}$ shift to negative direction is expected. The changes of the wound-potential are created by induced electric field (EF) and responsible for injury current (IC) as shown in Figure 1.

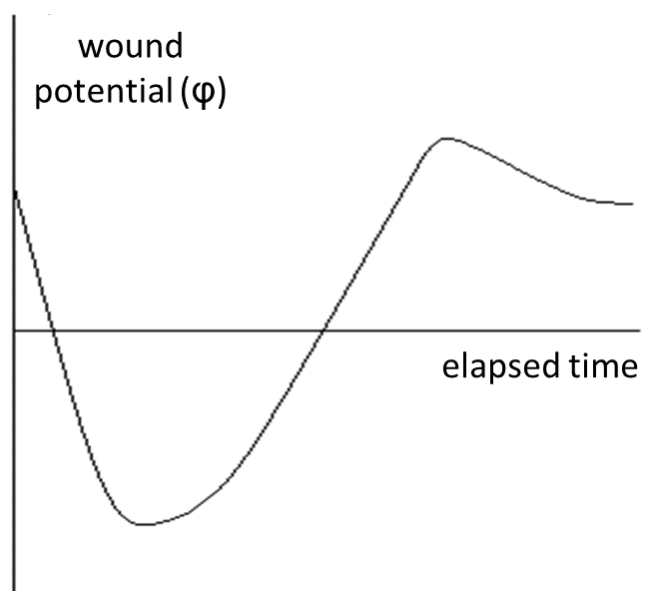

Figure 1. The potential of the wound $(\varphi)$ changes during the healing process, the homeostatic control regulates its development. The figure is only orienting the reader about the function of wound potential vs. elapsed time, the axes are shown with arbitrary units. 
The wound-healing starts with depolarization triggering the electro-kinetic electrolyte transport. The wound starts healing, the newly born cells (and the process produce them) utilize the electrolyte, and the remaining amount is cleared away by hyperpolarization. With this the wound-healing is finished. We expect similar effects in case of tumors.

The exchange of electrolytes by the capillary vessels is necessary to study for the action of hyperthermia. The capillary is $400-700 \mu \mathrm{m}$ length, $8-10 \mu \mathrm{m} \mathrm{di}$ ameter "tubes" having $0.5 \mu \mathrm{m}$ thick walls, forming by loose-fitting endothelial cells, Figure 2.

Two types of electrolyte exchanges are active between the capillaries and their environmental interstitial electrolyte. Both are osmotic interactions in opposite directions. The interstitial osmotic pressure is usually $-0.786 \mathrm{kPa}$, while the capillary pressure changes along the length of the tube: $3.3 \mathrm{kPa}$ at its beginning and $1.3 \mathrm{kPa}$ at its end. The osmotic pressure inside the capillary is $3.67 \mathrm{kPa}$, while in the interstitial space it is $0.65 \mathrm{kPa}$ [60]. (Other datasets [61] differ a little, but the basic conclusion could be developed.) The solved proteins mainly cause the osmotic pressure in the capillary in the electrolyte. Supposing ideal conditions, the flow of electrolyte is governed by the pressure differences deducting the osmotic pressure. The operating pressure at the beginning of the capillary is $\Delta p_{e}=(3.3-3.67)-(-0.786-0.65)=1.04 \mathrm{kPa}$, while at the end of the capillary $\Delta p_{e}=(1.3-3.67)-(-0.786-0.65)=-0.93 \mathrm{kPa}$. Consequently, the beginning of capillary has outflow, while its end has in-flow. The volume of the in/out flows is determined by the permeability of the capillary-wall. In normal cases the outflow is larger; the difference is balanced by the lymph system.

Growing temperature increases the osmotic pressure both in- and outside by

$$
\Delta \pi=\frac{\pi}{T} \Delta T
$$

Two centigrade temperature increase makes the growth of osmotic pressure in the capillary:

$$
\Delta \pi=\frac{\pi}{T} \Delta T=\frac{3.67}{310} \times 7=0.083 \mathrm{kPa}
$$

moreover, in the interstitial space:

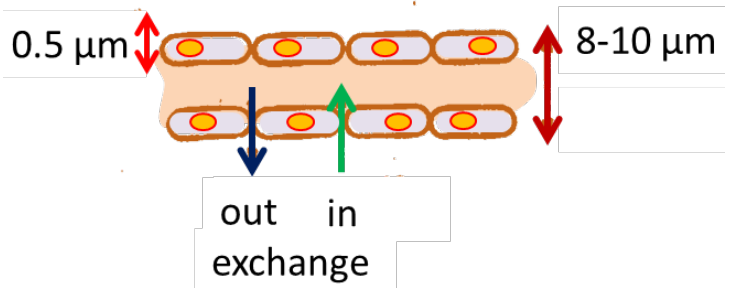

Figure 2. The capillary wall with loose-fitting endothelial cells is semi-permeable. The wall-thickness $(0.5 \mu \mathrm{m})$ and the lumen diameter $(8-10 \mu \mathrm{m})$ are shown, but the drawing is not in proportional scale. 


$$
\Delta \pi_{i}=\frac{\pi_{i}}{T} \Delta T=\frac{0.65}{310} \times 7=0.014 \mathrm{kPa}
$$

\section{Discussion}

The temperature increase reduces the outflow while enhances the volume of in-flow, by altering the osmotic pressure. Consequently, when nothing else happens, only local temperature changes in the target, the effusion should be decreased by growth temperature.

However, the opposite occurs when the protein and other ionic concentration grows in the interstitial fluid, so the immediate cellular distortion (lysis, necrosis) increases the drift which could support effusion. The necrotic cell-killing is a consequence of the conventional hyperthermia paradigm having a dose concept based on necrosis. The present conventional hyperthermia in oncology has doses and connected protocols based on necrotic damages [62]. The dosing problem is one of the main barriers to the wide clinical acceptance of hyperthermia in oncology [63]. The present dosing in hyperthermia is explained by the following principles:

1) Isothermal heating of the target trying to distribute the temperature as homogeneously as possible [64];

2) The inhomogeneity of the temperature in space is taken into account at the dosing explicitly in percentages [65];

3) Cumulative equivalent minutes (CEM) is the conventionally accepted dosing unit which is based on the Arrhenius principle [66] [67] [68];

4) Arrhenius plot is applied for the necrotic changes [69] [70]; and could change by the drugs administered in complementary chemotherapy [71];

5) The CEM-dose was introduced by in-vitro measurement of necrosis [72] [73];

6) CEM is applied in correlation of tumour-size (local control) [74] [75].

The CEM concept has various and serious disadvantages in the point of view of necrotic cell-death:

1) Results of CEM-dosing protocols correlate only slightly with clinical practice [76] [77] [78].

2) The CEM concept fails at higher temperatures of necrosis [79].

3) Like we showed above necrosis could change the microenvironment and the osmotic flow supports forming/promoting effusion.

The necrotic-distortion is not a single effect supporting the development of effusion in conventional oncologic hyperthermia. The increased vessel-wall permeability with the high temperature also supports the effusion. It became a considerable problem when the permeability increase reaches such merit that the large molecules (proteins) could pass through them, too. In this case, the internal osmotic pressure decreases gaining the out- and suppressing the in-flow in the capillary.

Consequently, high-temperature local hyperthermia supports the forming of effusion with the increase of permeability of vessels; which together with the 
above-discussed necrosis effect could cause that a tumor with effusion or larger electrolyte accumulation (e.g. ascites, pleural effusion) become contraindicated with conventional hyperthermia therapies.

The other problematic issue for conventional hyperthermia is that the wound has IC for apparent wound healing and it became permanent in case of tumors. IC directs the oriented cellular division and guides the cell-migration. We prove that the IC is connected to the volume-current of the electrolyte which always flows to the direction of the wound. The role of this extra flow is ensuring a part of the supply of nutrients for cellular demands at the processes of regeneration of the whole tissue. In the case of inflammation or even in a solid tumor similar process induces effusion. When hyperthermia increases the necrotic distortion making real wound at a tumor, the IC increases trigger more cell-proliferation than a tumor had before.

The additional effect to previous effects is triggered by the heat-flow of thermo-diffusion. When the temperature gradient is directed from the target to the neighboring tissues, it will induce thermodynamical force to eliminate the electrolyte from the target, re-establish the resting potential of the cells and limit the electrolyte flow caused by IC. Consequently, the well-focused hyperthermia could be advantageous to clear the effusion from the target. The problem is however that the isothermal concept heats the complete tumour-mass intending the same high temperature, triggering physiological feedback of thermal homeostasis. Intensive blood-flow starts supplying the tumour-cells with necessary nutrients for proliferation and the risk of invasion of malignant cell increases, their dissemination in the blood-flow and at the end forming and/or supporting metastases. Furthermore, conventional hyperthermia produces swelling of the cells, the growing cellular volume, and the forming micro-effusions are measurable in situ [80].

Hyperthermia is devoted to destroying the tumour-cells, but not necessarily with necrosis. The electric excitation could also have a role which is shown in the generalization of the CEM concept [81].

A gentle and at the same time more efficient way exists using the natural processes which are supported by hyperthermia. The challenge could be solved by dropping out the necrotic dose-reference $\left(43^{\circ} \mathrm{C}\right)$ and the isothermal (homogeneous) heating of the complete tumour-mass. If we used the natural inhomogeneity of the tissues, we would be able to select the malignant cells, even their parts which we could excite by an external field. This is the nano-heating technology, which is realized by the mEHT applications [11] [13]. The naturally present membrane rafts of malignant cells are selectively heated up [82], producing at least $3^{\circ} \mathrm{C}$ higher temperature on these nanoclusters of transmembrane proteins than their environment [83]. With this selective nano-heating, the micro-environmental effusion forming necrosis increase of vessel-wall permeability, as well as the rise of the wound triggered IC could be avoided. Consequently, the potential gradient decreases, so the IC will be smaller, too. The reduction of the IC accompanied by the lower flow-rate of the electrolyte is due to the Onsager's cross-effects, so the growth rate of effusion will also be reduced. 
This leads to a very useful application: the effusion-forming is not contraindicated for mEHT, it even could be applied to such effusion-sensitive organs like brain [84] [85]. This well applied therapeutic hyperthermia re-establishes the negative resting potential of the wound, and the thermal diffusion by the temperature gradient decreases the electrolyte transport supporting by the injury current. In the case of the large size of free electrolytes, however, the efficacy for selective tumour-heating could decrease due to the energy-absorption of the electrolyte, too.

The treatment of primary brain tumors (e.g. anaplastic astrocytoma and glioblastoma multiform) could be considered as early clinical proof of the method because hyperthermia likely causes cerebral edema which was not the case in the application of mEHT to advanced primary brain tumors. It is shown that $\mathrm{mEHT}$ is safe even for high-line (palliative care) treatments with high dose application [84]. This advantage is well mirrored in clinical trials of variously advanced brain tumors [85] [86] [87] [88]. The newest results show the effective curative treatment of mEHT in a prospective, randomized double-arm clinical trial, $(n=260)$ for peritoneal carcinomatosis. In this study, the mEHT together with traditional Chinese medicine (TCM) versus intraperitoneal chemo infusion (IPCI) were studied in the treatment of malignant ascites [89]. The combination of mEHT with TCM provided better control of peritoneal carcinomatosis than standard IPCI with less toxicity. In this trial, mEHT was applied 60 min per session every other day for 4 weeks, totally 14 sessions. TCM decoction was applied orally, $400 \mathrm{~mL}$ daily (its composition is described in the study [89] in details). The occlusive IPCI with cisplatin (30 - $60 \mathrm{mg}$ ) and fluorouracil $\left(500-600 \mathrm{mg} / \mathrm{m}^{2}\right)$ was applied two times, biweekly. No case was lost or excluded during the trial so all patients were treated and analyzed. Results of the treatments were evaluated one month after the treatment in both groups. Objective response rate in $\mathrm{mEHT}$ combined arm of the study was $77.69 \%(101 / 130)$ vs. $63.85 \%(73 / 130)$ in the IPCI group $(\mathrm{p}<0.05)$. The quality of life in the combined group was $49.23 \%$ vs. $32.3 \%$ in IPCI group $(\mathrm{p}<0.05)$. All adverse effects were grade I, and by the distribution in combined group was $2.3 \%(3 / 130)$ vs. $12.3 \%(16 / 130)$ in IPCI group $(\mathrm{p}<0.05)$.

The limitations of the present clinical application of $\mathrm{mEHT}$ are the cases with large free electrolyte volumes in the body without proper drain. Furthermore, the urinary bladder, stomach and other cavities which contain large volume of free electrolyte have to be emptied before the treatment in the area.

\section{Conclusion}

Forming of effusion is a complex process, regulated by multiple effects under homeostatic control. The necrosis-based isothermal hyperthermia approach could stimulate and growth further the effusion and other free electrolytes (e.g. ascites, pleural effusion), contrary to the selective heterogenic (non-isothermal) $\mathrm{mEHT}$, which makes mild complete heating while heats extremely the chosen aggregates of transmembrane proteins. It is safe to treat any free-electrolyte lesions, too. Fur- 
ther investigation in direction to such sensitive organs like brain, when intracranial pressure could be life threatening by heating, is in progress.

\section{Acknowledgements}

This work was supported by the Hungarian Competitiveness and Excellence Programme grant (NVKP_16-1-2016-0042).

\section{References}

[1] Grannis Jr, F.W., Kim, J.Y. and Lay, L. (2017) Fluid Complications. Cancer Network. http://www.cancernetwork.com

[2] Isikawa, T., Kokura, S., Sakamoto, N., et al. (2012) Phase II Trial of Combined Regional Hyperthermia and Gemcitabine for Locally Advanced or Metastatic Pancreatic Cancer. International Journal of Hyperthermia, 28, 597-604. https://doi.org/10.3109/02656736.2012.695428

[3] Sharma, H.S. (2006) Hyperthermia Induced Brain Oedema: Current Status \& Future Perspectives. Indian Journal of Medical Research, 123, 629-652.

[4] Seegenschmiedt, M.H., Fessenden, P. and Vernon, C.C. (1996) Thermoradiotherapy and Thermochemotherapy. Springer Verlag, Berlin Heidelberg, 1-2.

[5] Kosaka, M., Sugahara, T., Schmidt, K.L., et al. (2001) Thermotherapy for Neoplasia, Inflammation, and Pain. Springer Verlag, Tokyo.

[6] Mórocz, I.A., Hynynen, K., Gudbjartsson, H., Peled, S., Colucci, V. and Jólesz, F.A. (1998) Brain Edema Development after MRI Guided Focused Ultrasound Treatment. Journal of Magnetic Resonance Imaging, 8, 136-142.

https://doi.org/10.1002/jmri.1880080126

[7] Hosotani, K., Katsumura, H., Kabuto, M., Handa, Y., Kubota, T. and Hayashji, M. (1993) Effect of Whole-Body Hyperthermia on the Development of Peritumoral Brain Oedema. International Journal of Hyperthermia, 1, 25-36. https://doi.org/10.3109/02656739309061476

[8] Lyons, B.E., Britt, R.H. and Strohbehn, J.W. (1984) Localized Hyperthermia in the Treatment of Malignant Brain Tumors Using an Interstitial Microwave Antenna Array. IEEE Transactions on Biomedical Engineering, 31, 53-62. https://doi.org/10.1109/TBME.1984.325370

[9] Gilly, F.N., Carry, P.Y., Bracket, A., et al. (1992) Treatment of Malignant Peritoneal Effusion in Digestive and Ovarian Cancer. Medical Oncology and Tumor Pharmacotherapy, 9, 177-181.

[10] Neuwirth, M.G., Alexander, H.R. and Karakousis, G.C. (2015) Then and Now: Cytoreductive Surgery with Hyperthermic Intraperitoneal Chemotherapy (HIPEC), a Historical Perspective. Journal of Gastrointestinal Oncology, 7, 18-28.

[11] Szasz, A., Szasz, N. and Szasz, O. (2010) Oncothermia-Principles and Practices. Springer Science, Heidelberg.

[12] Andocs, G., Rehman, M.U., Zhao, Q.-L., Tabuchi, Y., Kanamori, M. and Kondo, T. (2016) Comparison of Biological Effects of Modulated Electro-Hyperthermia and Conventional Heat Treatment in Human Lymphoma U937 Cell. Cell Death Discovery (Nature Publishing Group), 2, Article ID: 16039. https://doi.org/10.1038/cddiscovery.2016.39

[13] Szasz, O. and Szasz, A. (2014) Oncothermia-Nano-Heating Paradigm. Journal of Cancer Science and Therapy, 6, 117-121. https://doi.org/10.4172/1948-5956.1000259 
[14] Andocs, G., Renner, H., Balogh, L., Fonyad, L., Jakab, C. and Szasz, A. (2009) Strong Synergy of Heat and Modulated Electro-Magnetic Field in Tumor Cell Killing, Study of HT29 Xenograft Tumors in a Nude Mice Model. Strahlentherapie und Onkologie, 185, 120-126. https://doi.org/10.1007/s00066-009-1903-1

[15] Szasz, A., Iluri, N. and Szasz, O. (2013) Local Hyperthermia in Oncology-To Choose or Not to Choose? In: Huilgol, N., Ed., Hyperthermia, InTech, Rijeka.

[16] West, J. (2012) Respiratory Physiology: The Essentials. 9th Edition, Lippincott Williams \& Wilkins, Baltimore, 177.

[17] Levick, J.R. (2003) Introduction to Cardiovascular Physiology. Oxford Press, Oxford, $179-180$.

[18] Huxley, V.H. and Scallan, J. (2011) Lymphatic Fluid: Exchange Mechanisms and Regulation. The Journal of Physiology, 589, 2935-2943. https://doi.org/10.1113/jphysiol.2011.208298

[19] De Luca Jr, L.A., David, R.B. and Menani, J.V. (2014) Homeostasis and Body Fluid Regulation, Neurobiology of Body Fluid Homeostasis. In: De Luca, L.A., Menani, J.V. and Johnson, A.K., Eds., Transduction and Integration, CRC Press/Taylor \& Francis, Boca Raton, Ch. 15.

[20] Levick, J.R. and Michel, C.C. (2010) Microvascular Fluid Exchange and the Revised Starling Principle. Cardiovascular Research, 87, 198-210. https://doi.org/10.1093/cvr/cvq062

[21] Woodcock, T.E. and Woodcock, T.M. (2012) Revised Starling Equation and the Glycocalyx Model of Transvascular Fluid Exchange: An Improved Paradigm for Prescribing Intravenous Fluid Therapy. British Journal of Anaesthesia, 108, 384-394. https://doi.org/10.1093/bja/aer515

[22] Carbon, M., Wübbeler, G., Mackert, B.-M., et al. (2004) Non-Invasive Magnetic Detection of Human Injury Currents. Clinical Neurophysiology, 115, 1027-1032. https://doi.org/10.1016/j.clinph.2003.12.035

[23] McCaig, C.D., Rajnicek, A.M., Song, B. and Zhao, M. (2005) Controlling Cell Behaviour Electrically: Current Views and Future Potential. Physiological Reviews, 85, 943-978. https://doi.org/10.1152/physrev.00020.2004

[24] Barker, A.T., Jaffe, L.F. and Vanable Jr, J.W. (1982) The Glabrous Epidermis of Cavies Contains a Powerful Battery. American Journal of Physiology, 242, 358-366.

[25] Reid, B., McCaig, C.D., Zhao, M., et al. (2005) Wound Healing in Rat Cornea: The Role of Electric Currents. FASEB Journal, 19, 379-386. https://doi.org/10.1096/fj.04-2325com

[26] Reid, B., Nuccitelli, R. and Zhao, M. (2007) Non-Invasive Measurement of Bioelectric Currents with a Vibrating Probe. Nature Protocols, 2, 661-669. https://doi.org/10.1038/nprot.2007.91

[27] Mackert, B.-M., Mackert, J., Wübbeler, G., et al. (1999) Magnetometry of Injury Currents from Human Nerve and Muscle Specimens Using Superconducting Quantum Interferences Devices. Neuroscience Letters, 262, 163-166. https://doi.org/10.1016/S0304-3940(99)00067-1

[28] Song, B., Zhao, M., Forrester, J.V., et al. (2002) Electrical Cues Regulate the Orientation and Frequency of Cell Division and the Rate of Wound Healing In Vivo. PNAS, 99, 13577-13582. https://doi.org/10.1073/pnas.202235299

[29] Chiang, M.C., Cragoe Jr, E.J. and Vanable Jr., J.W. (1991) Intrinsic Electric Fields Promote Epithelization of Wounds in the Newt, Notophthalmus viridescens. Developmental Biology, 146, 377-85. https://doi.org/10.1016/0012-1606(91)90239-Y 
[30] Zhao, M. (2009) Electrical Fields in Wound Healing-An Overriding Signal That Directs Cell Migration. Seminars in Cell and Developmental Biology, 20, 674-682. https://doi.org/10.1016/j.semcdb.2008.12.009

[31] Ud-Din, S., Sebastian, A., Giddings, P., Colthurst, J., Whiteside, S., Morris, J., et al. (2015) Angiogenesis Is Induced and Wound Size Is Reduced by Electrical Stimulation in an Acute Wound Healing Model in Human Skin. PLOS ONE, 10, e0124502.

[32] Katchalsky, A. and Curran, P. (1967) Non-Equilibrium Thermodynamics in Biophysics. Harvard University Press, Cambridge-Massachusets.

[33] Scallan, J., Huxley, V.H. and Korthuis, R.J. (2010) Capillary Fluid Exchange: Regulation, Functions, and Pathology. In: Pathophysiology of Edema Formation, Morgan \& Claypool Life Sciences, San Rafael, CA, Chapter 4.

[34] Nordenstrom, B.W.E. (1983) Biologically Closed Electric Circuits: Clinical Experimental and Theoretical Evidence for an Additional Circulatory System. Nordic Medical Publications, Stockholm.

[35] Nordenstrom, B.W.E. (1998) Exploring BCEC-Systems, (Biologically Closed Electric Circuits). Nordic Medical Publications, Stockholm.

[36] Nordenstrom, B.W.E. (1992) Impact of Biologically Closed Electric Circuits (BCEC) on Structure and Function. Integrative Physiological and Behavioral Science, 27, 285-303. https://doi.org/10.1007/BF02691165

[37] Mycielska, M.E. and Djamgoz, M.B.A. (2004) Cellular Mechanisms of Direct-Current Electric Field Effects: Galvanotaxis and Metastatic Disease. Journal of Cell Science, 117, 1631-1639. https://doi.org/10.1242/jcs.01125

[38] Pu, J., McCaig, C.D., Cao, L., et al. (2007) EGF Receptor Signalling Is Essential for Electric-Field-Directed Migration of Breast Cancer Cells. Journal of Cell Science, 120, 3395-3403. https://doi.org/10.1242/jcs.002774

[39] Nordenström, B.E.W. (1978) Preliminary Clinical Trials of Electrophoretic Ionization in the Treatment of Malignant Tumors. IRCS Journal of Medical Science, 6 , 537-540.

[40] Nordenström, B.E.W. (1985) Electrochemical Treatment of Cancer. Annales De Radiologie, 28, 128-129.

[41] Watson, B.W. (1991) Reappraisal: The Treatment of Tumors with Direct Electric Current. Medical Science Research, 19, 103-105.

[42] Samuelsson, L., Jonsson, L. and Stahl, E. (1983) Percutaneous Treatment of Pulmonary Tumors by Electrolysis. Radiologie, 23, 284-287

[43] Miklavcic, D., Sersa, G., Kryzanowski, M., et al. (1993) Tumor Treatment by Direct Electric Current, Tumor Temperature and $\mathrm{pH}$, Electrode Materials and Configuration. Bioelectrochemistry and Bioenergetics, 30, 209-220. https://doi.org/10.1016/0302-4598(93)80080-E

[44] Nordenstrom, B.W.E. (2009) Biologically Closed Electric Circuits: Activation of Vascular Interstitial Closed Electric Circuits for Treatment of Inoperable Cancers. Journal of Bioelectricity, 3, 137-154. https://doi.org/10.1080/15368378409035964

[45] Nordenstrom, B.W.E. (1994) The Paradigm of Biologically Closed Electric Circuits (BCEC) and the Formation of an International Association (IABC) for BCEC Systems. The European Journal of Surgery. Supplement, 574, 7-23.

[46] Dvorak, H.F. (1986) Tumors: Wounds That Do Not Heal. Similarities between Tumor Stroma Generation and Wound Healing. The New England Journal of Medicine, 315, 1650-1659. https://doi.org/10.1056/NEJM198612253152606

[47] Schäfer, M. and Werner, S. (2008) Cancer as an Overhealing Wound: An Old Hy- 
pothesis Revisited. Nature Reviews Molecular Cell Biology, 9, 628-638. https://doi.org/10.1038/nrm2455

[48] Singh, K. (2015) Carcinogenesis and Diabetic Wound Healing: Evidences of Parallelism. Current Diabetes Reviews, 11, 32-45. https://doi.org/10.2174/1573399811666150109122205

[49] Goh, J. and Ladiges, W.C. (2014) Exercise Enhances Wound Healing and Prevents Cancer Progression during Aging by Targeting Macrophage Polarity. Mechanisms of Ageing and Development, 39, 41-48. https://doi.org/10.1016/j.mad.2014.06.004

[50] Meng, X. and Riordan, N.H. (2006) Cancer Is a Functional Repair Tissue. Medical Hypotheses, 66, 486-490. https://doi.org/10.1016/j.mehy.2005.09.041

[51] Kimberly, M.A., Opdenaker, L.M., Flynn, D. and Sims-Mourtada, J. (2015) Wound Healing and Cancer Stem Cells: Inflammation as a Driver of Treatment Resistance in Breast Cancer. Cancer Growth Metastasis, 8, 1-13.

[52] Rybinski, B., Franco-Barraza, J. and Cukierman, E. (2014) The Wound Healing, Chronic Fibrosis, and Cancer Progression Triad. Physiological Genomics, 46, 223-244. https://doi.org/10.1152/physiolgenomics.00158.2013

[53] Calvo, F., Randfl, R., Hooper, S., Faruggia, A.G., Moeendarbary, E., Bruckbauer, A., Batista, F., Charras, G. and Sahai, E. (2015) Cdc42EP3/BORG2 and Septin Network Enables Mechano-Transduction and the Emergence of Cancer Associated Fibroblasts. Cell Reports, 13, 1-16. https://doi.org/10.1016/j.celrep.2015.11.052

[54] Kaspera, M., Jaksa, V., Area, A., Bergströma, Å., Schwägera, A., Svärda, J., Teglunda, S., Barkerc, N. and Toftgård, R. (2011) Wounding Enhances Epidermal Tumorigenesis by Recruiting Hair Follicle Keratinocytes. PNAS, 108, 4099-4104. https://doi.org/10.1073/pnas.1014489108

[55] Wolf, A.A. (1981) On a Unified Theory of Cancer Etiology and Treatment Based on the Superconduction Double-Dipole Model. Physiological Chemistry and Physics, 13, 493-510.

[56] Giaquinta, G., Di Mauro, C., Onori, S. and Cannistraro, S. (1985) Experimental Evidence of Superconducting Properties in Human Ceruloplasmin. In: Gandolfo, G., Michaelson, S.M. and Rindi, A., Eds., Biological Effects and Dosimetry of Static and ELF Electromagnetic Fields, Springer, Berlin, 339-343. https://doi.org/10.1007/978-1-4613-2099-9_21

[57] Calvo, F.N. (2014) Tumor Microenvironment: Unleashing Metalloproteinases to Induce a CAF Phenotype. Current Biology, 24, No 20.

[58] Fierheller, M. and Sibbald, R.G. (2010) A Clinical Investigation into the Relationship between Increased Periwound Skin Temperature and Local Wound Infection in Patients with Chronic Leg Ulcers. Advances in Skin \& Wound Care, 23, 369-739. https://doi.org/10.1097/01.ASW.0000383197.28192.98

[59] Song, B., Zhao, M., Forrester, J., et al. (2004) Nerve Regeneration and Wound Healing Are Stimulated and Directed by an Endogenous Electrical Field In Vivo. Journal of Cell Science, 117, 4681-4690. https://doi.org/10.1242/jcs.01341

[60] Guyton, A.C. (1976) Textbook of Medical Physiology. Saunders, Philadelphia.

[61] Boron, W.F. (2004) A Cellular and Molecular Approach. Elsevier/Saunders, Philadelphia.

[62] Dewhirst, M.W., Viglianti, B.L., Lora-Michiels, M., et al. (2003) Basic Principles of Thermal Dosimetry and Thermal Thresholds for Tissue Damage from Hyperthermia. International Journal of Hyperthermia, 19, 267-294.

https://doi.org/10.1080/0265673031000119006 
[63] Jones, E., Thrall, D., Dewhirst, M.W. and Vujaskovic, Z. (2006) Prospective Thermal Dosimetry: The Key to Hyperthermia's Future. International Journal of Hyperthermia, 22, 247-253. https://doi.org/10.1080/02656730600765072

[64] Thrall, D.E., Rosner, G.L., Azuma, C., Larue, S.M., Case, B.C., Samulsky, T. and Dewhirst, M.W. (2000) Using Units of CEM43oC T90 Local Hyperthermia Thermal dose Can Be Delivered as Prescribed. International Journal of Hyperthermia, 16, 415-428. https://doi.org/10.1080/026567300416712

[65] Leophold, K.A., Dewhirst, M.W., Samulsky, T.V., Dodge, R.K., Georg, S.L., Blivin, J.L., Prosnitz, L.R. and Oleson, J.R. (1993) Cumulative Minutes with T90 Greater Than Temindex Is Predictive of Response of Superficial Malignancies to Hyperthermia and Radiation. International Journal of Radiation Oncology ${ }^{\star}$ Biology Physics, 25, 841-847. https://doi.org/10.1016/0360-3016(93)90314-L

[66] Dewey, W.C. (1994) Arrhenius Relationships from the Molecule and Cell to the Clinic. International Journal of Hyperthermia, 10, 457-483. https://doi.org/10.3109/02656739409009351

[67] Dewey, W.C., Hopwood, L.E., Sapareto, S.A. and Gerweck, L.E. (1977) Cellular Response to Combination of Hyperthermia and Radiation. Radiology, 123, 463-474. https://doi.org/10.1148/123.2.463

[68] Sapareto, S.A. and Dewey, W.C. (1984) Thermal Dose Determination in Cancer Therapy. International Journal of Radiation Oncology ${ }^{\star}$ Biology ${ }^{\star}$ Physics, 10, 787-800. https://doi.org/10.1016/0360-3016(84)90379-1

[69] Moritz, A.R. and Henriques, F.C. (1947) Studies of Thermal Injury. ii. The relative importance of Time and Surface Temperature in the Causation of Cutaneous Burns. American Journal of Pathology, 23, 695-720.

[70] Henriques, F.C. (1947) Studies of Thermal Injury v. The Predictability and the Significance of Thermally Induced Rate Processes Leading to Irreversible Epidermal Injury. Archives of Pathology, 43, 489- 502.

[71] Urano, M. and Douple, E. (1994) Chemopotentiation by Hyperthermia. Hyperthermia in Oncology, 4, 173.

[72] Sapareto, S.A. and Dewey, W.C. (1984) Thermal Dose Determination in Cancer Therapy. International Journal of Radiation Oncology ${ }^{\star}$ Biology ${ }^{\star}$ Physics, 10, 787-800. https://doi.org/10.1016/0360-3016(84)90379-1

[73] Pearce, J.A. (2009) Relationship between Arrhenius Models of Thermal Damage and the CEM 43 Thermal Dose. Energy-Based Treatment of Tissue and Assessment $V$, 7181, Article ID: 718104. https://doi.org/10.1117/12.807999

[74] Perez, C.A. and Sapareto, S.A. (1984) Thermal Dose Expression in Clinical Hyperthermia and Correlation with Tumor Response/Control. Cancer Research, 44, 4818-4825.

[75] Thrall, D.E., LaRue, S.M., Yu, D., Samulski, T., Sanders, L., Case, B., Rosner, G., Azuma, C., Poulson, J., Pruitt, A.F., Stanley, W., Hauck, M.L., Williams, L., Hess, P. and Dewhirst, M.W. (2005): Thermal Dose Is Related to Duration of Local Control in Canine Sarcomas Treated with Thermoradiotherapy. Clinical Cancer Research, 11, 5206-5214. https://doi.org/10.1158/1078-0432.CCR-05-0091

[76] Maguire, P.D., et al. (2001) A Phase II Trial Testing the Thermal Dose Parameter CEM43oCT90 as a Predictor of Response in Soft Tissue Sacomas Treated with Pre-Operative Thermorasiotherapy. International Journal of Hyperthermia, 17, 283-290. https://doi.org/10.1080/02656730110039449

[77] Dewhirst, M.W., Vujaskovic, Z., Jones, E. and Thrall, D. (2005) Re-Setting the Biologic Rationale for Thermal Therapy. International Journal of Hyperthermia, 21, 
779-790. https://doi.org/10.1080/02656730500271668

[78] de Bruijne, M., van der Holt, B., van Rhoon, G.C., et al. (2010) Evaluation of CEM $43^{\circ} \mathrm{CT} 90$ Thermal Dose in Superficial Hyperthermia; A Retrospective Analysis. Strahlentherapy \& Onkologie (Radiotherapy and Oncology), 186, 436-443. https://doi.org/10.1007/s00066-010-2146-x

[79] Assi, H. (2009) A New cem43 Thermal Dose Model Based on Vogel-TammannFulcher Behavior in Thermal Damage Processes. Ryerson University, Toronto, Ontario.

[80] Esrick, M.A. and McRae, D.A. (1994) The Effect of Hyperthermia Induced Tissue Conductivity Changes on Electrical Impedance Temperature Mapping. Physics in Medicine \& Biology, 39, 133-144. https://doi.org/10.1088/0031-9155/39/1/008

[81] Vincze, Gy., Szasz, O. and Szasz, A. (2015) Generalization of the Thermal Dose of Hyperthermia in Oncology. Open Journal of Biophysics, 5, 97-114. https://doi.org/10.4236/ojbiphy.2015.54009

[82] Vincze, Gy., Szigeti, Gy., Andocs, G. and Szasz, A. (2015) Nanoheating without Artificial Nanoparticles. Biology and Medicine, 7, 249.

[83] Andocs, G., Rehman, M.U., Zhao, Q.L., Papp, E., Kondo, T. and Szasz, A. (2015) Nanoheating without Artificial Nanoparticles Part II. Experimental Support of the Nanoheating Concept of the Modulated Electro-Hyperthermia Method, Using U937 Cell Suspension Model. Biology and Medicine, 7, 1-9. https://doi.org/10.4172/0974-8369.1000247

[84] Wismeth, C., Dudel, C., Pascher, C., Ramm, P., Pietsch, T., Hirschmann, B., Reinert, C., Proescholdt, M., Rümmele, P., Schuierer, G., Bogdahn, U. and Hau, P. (2010) Transcranial Electro-Hyperthermia Combined with Alkylating Chemotherapy in Patients with Relapsed High-Grade Gliomas-Phase I Clinical Results. Journal of Neuro-Oncology, 98, 395-405. https://doi.org/10.1007/s11060-009-0093-0

[85] Sahinbas, H., Groenemeyer, D.H.W., Boecher, E. and Szasz, A. (2007) Retrospective Clinical Study of Adjuvant Electro-Hyperthermia Treatment for Advanced Brain-Gliomas. Deutsche Zeitschrift fuer Onkologie, 39, 154-160. https://doi.org/10.1055/s-2007-986020

[86] Fiorentini, G., Giovanis, P., Rossi, S., Dentico, P., Paola, R., Turrisi, G. and Bernardeschi, P. (2006) A Phase II Clinical Study on Relapsed Malignant Gliomas Treated with Electro-Hyperthermia. In Vivo, 20, 721-724.

[87] Hager, E.D., Sahinbas, H., Groenemeyer, D.H. and Migeod, F. (2008) Prospective Phase II Trial for Recurrent High-Grade Malignant Gliomas with Capacitive Coupled Low Radiofrequency (LRF) Deep Hyperthermia. ASCO. Journal of Clinical Oncology, Annual Meeting Proceedings (Post-Meeting Edition), 26, 2047.

[88] Hager, E.D. and Birkenmeier, J. (2006) Glioblastoma multiforme Grad IV: Regionale Tiefenhyperthermie, Antiangiogenese mit Thalidomid, Hochdosis-Ascorbinsäureinfusionen und komplementäre Therapie. Deutsche Zeitschrift für Onkologie, 38, 133-135. https://doi.org/10.1055/s-2006-952050

[89] Pang, C.L.K., Xinting, Z., Zhen, W., Junwen, O., Yimin, L., Roussakow, R., et al. (2017) Local Modulated Electro-Hyperthermia in Combination with Traditional Chinese Medicine vs. Intraperitoneal Chemoinfusion for Treatment of Peritoneal Carciomatosis with Malignant Ascites: A Phase II Randomized Trial. Molecular and Clinical Oncology, 6, 723-732. https://doi.org/10.3892/mco.2017.1221 